\title{
Corrigendum: Draft genome sequence and genetic transformation of the oleaginous alga Nannochloropsis gaditana
}

Randor Radakovits, Robert E. Jinkerson, Susan I. Fuerstenberg, Hongseok Tae, Robert E. Settlage, Jeffrey L. Boore \& Matthew C. Posewitz

Nature Communications 3:686 doi: 10.1038/ncomms1688 (2012); Published 21 Feb 2012; Updated 19 Sep 2013

The original version of this Article contained an error in the spelling of Nannochloropsis in the title of the paper. This has now been corrected in both the PDF and HTML versions of the Article. 